Supplement of Atmos. Chem. Phys. Discuss., 15, 26423-26479, 2015

http://www.atmos-chem-phys-discuss.net/15/26423/2015/

doi:10.5194/acpd-15-26423-2015-supplement

(C) Author(s) 2015. CC Attribution 3.0 License.

(c) (i) Valley urban-industrial environment

\author{
N. Amil et al. \\ Correspondence to: M. T. Latif (talib@ukm.my)
}

The copyright of individual parts of the supplement might differ from the CC-BY 3.0 licence. 


\section{Experimental quality assurance and quality control (QA/QC)}

2 For filter sampling, the elapsed time indicator (ETI) as well as the flow/pressure recorder 3 chart was operated and checked daily to ensure the quality of sampling. Filter weighing was 4 repeated three times and the average of those three were used for calculation. $\mathrm{PM}_{2.5}$ mass

5 were corrected by deducting the monthly filter blank (FB).

6

7 Anion and cation analysis: Using ion chromatography (IC), it was ensured that all ion 8 regression coefficients were better than 0.999 before the sample analysis was begun and that 9 every 15th sample, the QA/QC samples were run, i.e. the ultra-pure water (UPW) and $1 \mathrm{ppm}$ standard prepared the same manner as the samples. These were used as validation tests for the method and the recovery purpose. All lab ware used in the ion analyses was Class A glassware.

Trace element analysis: Using inductively coupled plasma mass spectrometry (ICPMS), it was ensured that all element regression coefficients were better than 0.999 before the sample analysis was begun and that every 15 th sample, the QA/QC were samples run, i.e. the UPW, 1 ppm multi-element standard and standard reference material (SRM1648a). The reference material was $10 \mathrm{mg}$ of SRM1648a Urban Particulate Matter obtained from NIST (National Institute of Standards and Technology, MD, USA) while 1 ppm Multi-Element Calibration Standard 3 (Perkin Elmer Pure Plus, Perkin-Elmer; USA) both prepared the same manner as the samples to test the recovery and validation of the method. All lab ware used for trace element analyses was Teflon material, except for the syringes. 
1 Table S1. Descriptive statistics of experimental data (meteorological, gaseous and ion parameters); unit: mean \pm std (min - max). Remarks:

$2 \mathrm{ud}=$ undetected (below detection limit); $\mathrm{SIA}=$ secondary inorganic aerosol; $\mathrm{NR}=$ neutralisation ratio $=\left[\mathrm{NH}_{4}{ }^{+}\right] /\left(\left[\mathrm{SO}_{4}{ }^{2-}\right]+\left[\mathrm{NO}_{3}{ }^{-}\right]\right)\left(\mathrm{Squizzato} \mathrm{et}^{2}\right.$ 3 al., 2013); $\mathrm{SO}_{2}$ gas was converted from ppm to $\mu \mathrm{g} \mathrm{m}^{-3}$ assuming $1 \mathrm{ppm}=2619 \mu \mathrm{g} \mathrm{m}^{-3}\left(25^{\circ} \mathrm{C}, 1 \mathrm{~atm}\right)$.

\begin{tabular}{|c|c|c|c|c|}
\hline Elements & Unit & $\begin{array}{c}\text { ANNUAL } \\
\begin{aligned} 5 \text { Aug } 2011-18 \text { July } 2012 \\
n=81\end{aligned} \\
\end{array}$ & $\begin{array}{c}\text { SW } \\
15 \text { May - } 14 \text { Sept } \\
n=29 \\
\end{array}$ & $\begin{array}{c}\text { INT.2 } \\
15 \text { Sept }-30 \text { Oct } \\
n=7 \\
\end{array}$ \\
\hline API & - & $50 \pm 16(29-127)$ & $60 \pm 21(36-127)$ & $49 \pm 6(40-59)$ \\
\hline $\mathrm{T}$ & ${ }^{\circ} \mathrm{C}$ & $28.49 \pm 1.19(26.05-31.55)$ & $28.88 \pm 1.36(26.4-31.55)$ & $28.53 \pm 1.2(27.1-30.4)$ \\
\hline RH & $\%$ & $71.23 \pm 7.91(50.42-86.67)$ & $68.23 \pm 9.22(50.42-86.67)$ & $72.88 \pm 8.5(59.67-82.67)$ \\
\hline WS & $\mathrm{ms}^{-1}$ & $1.29 \pm 0.19(0.87-1.77)$ & $1.39 \pm 0.19(0.97-1.77)$ & $1.25 \pm 0.2(1.01-1.53)$ \\
\hline WD & Degree & $128.83 \pm 31.64(23.13-208.21)$ & $123.32 \pm 37.99(23.13-205)$ & $128.52 \pm 22.02(100-167.42)$ \\
\hline Rainfall & $\mathrm{mm}$ & $10.37 \pm 17.51(0-85.4)$ & $6.27 \pm 10.63(0-34.2)$ & $8.46 \pm 16.88(0-45.4)$ \\
\hline $\mathrm{CO}$ & ppm & $1.29 \pm 0.31(0.61-2.16)$ & $1.26 \pm 0.32(0.61-1.99)$ & $1.43 \pm 0.32(1.1-1.93)$ \\
\hline $\mathrm{O}_{3}$ & $\mathrm{ppm}$ & $0.01 \pm 0.01(\mathrm{ud}-0.03)$ & $0.01 \pm 0.01(\mathrm{ud}-0.02)$ & $0.02 \pm 0.01(0.01-0.03)$ \\
\hline $\mathrm{SO}_{2}$ & ppm & $0 \pm 0(\mathrm{ud}-0.01)$ & $0 \pm 0(\mathrm{ud}-0.01)$ & $0 \pm 0($ ud -0.01$)$ \\
\hline $\mathrm{NO}_{\mathrm{X}}$ & ppm & $0.06 \pm 0.01(0.03-0.11)$ & $0.06 \pm 0.01(0.03-0.08)$ & $0.07 \pm 0.01(0.06-0.09)$ \\
\hline NO & ppm & $0.03 \pm 0.01(0.01-0.07)$ & $0.03 \pm 0.01(0.01-0.04)$ & $0.03 \pm 0.01(0.02-0.05)$ \\
\hline $\mathrm{NO}_{2}$ & ppm & $0.03 \pm 0.01(0.02-0.05)$ & $0.03 \pm 0.01(0.02-0.05)$ & $0.04 \pm 0.01(0.03-0.05)$ \\
\hline $\mathrm{SO}_{4}{ }^{2-}$ & $\mu \mathrm{g} \mathrm{m}^{-3}$ & $1.33 \pm 0.88$ & $1.77 \pm 1.16$ & $1.62 \pm 0.78$ \\
\hline $\mathrm{ss}^{-\mathrm{SO}_{4}}{ }^{2-}$ & $\mu \mathrm{g} \mathrm{m}^{-3}$ & $0.08 \pm 0.09$ & $0.06 \pm 0.02$ & $0.02 \pm 0.01$ \\
\hline nss- $\mathrm{SO}_{4}{ }^{2-}$ & $\mu \mathrm{g} \mathrm{m}^{-3}$ & $1.29 \pm 0.9$ & $1.75 \pm 1.18$ & $1.61 \pm 0.79$ \\
\hline $\mathrm{NO}_{3}^{-}$ & $\mu \mathrm{g} \mathrm{m}^{-3}$ & $0.21 \pm 0.13$ & $0.19 \pm 0.08$ & $0.29 \pm 0.22$ \\
\hline $\mathrm{NH}_{4}^{+}$ & $\mu \mathrm{g} \mathrm{m}^{-3}$ & $0.99 \pm 0.85$ & $1.45 \pm 1.18$ & $1 \pm 0.64$ \\
\hline SIA & $\mu \mathrm{g} \mathrm{m}^{-3}$ & $2.4 \pm 1.68$ & $3.28 \pm 2.25$ & $2.78 \pm 1.58$ \\
\hline $\mathrm{SIA} / \mathrm{PM}_{2.5}$ & $\%$ & $8.54 \pm 2.97$ & $8.69 \pm 3.43$ & $9.62 \pm 2.96$ \\
\hline NR & - & $0.65 \pm 0.31$ & $0.72 \pm 0.19$ & $0.55 \pm 0.07$ \\
\hline $\mathrm{SO}_{4}{ }^{2}-\mathrm{SO}_{2}$ & $\mu \mathrm{g} \mathrm{m}^{-3}$ & $1.33-8.19$ & $1.77-9.5$ & $1.62-10.84$ \\
\hline
\end{tabular}




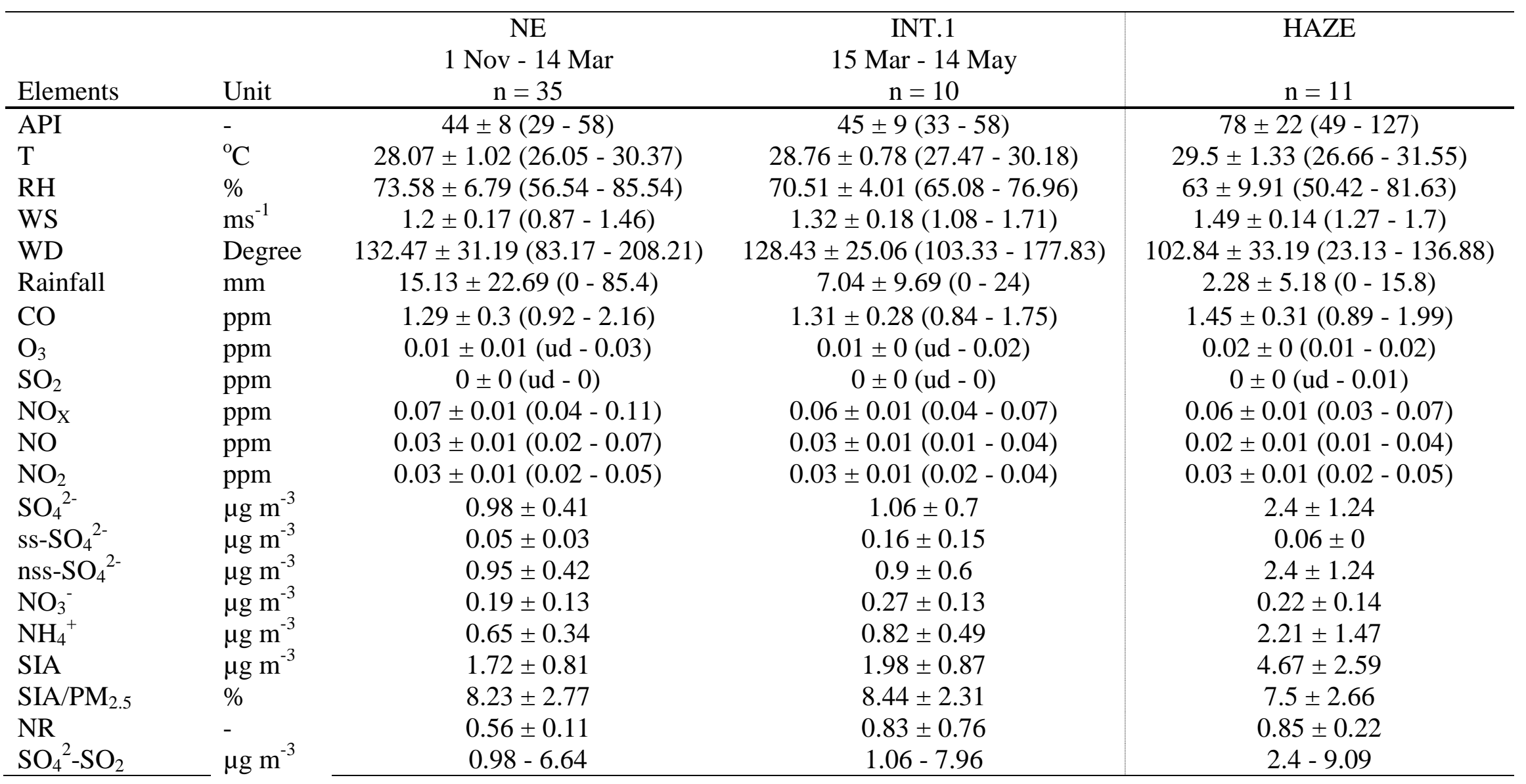


1 Table S2. Statistical compositions of $\mathrm{PM}_{2.5}$ during the one-year period including \% recovery

2 for anions and cations using $1 \mathrm{ppm}$ standard of single cation/anion standards; and for trace

3 element using standard reference material (SRM1648a). Remarks: MDL = method detection

4 limit

5

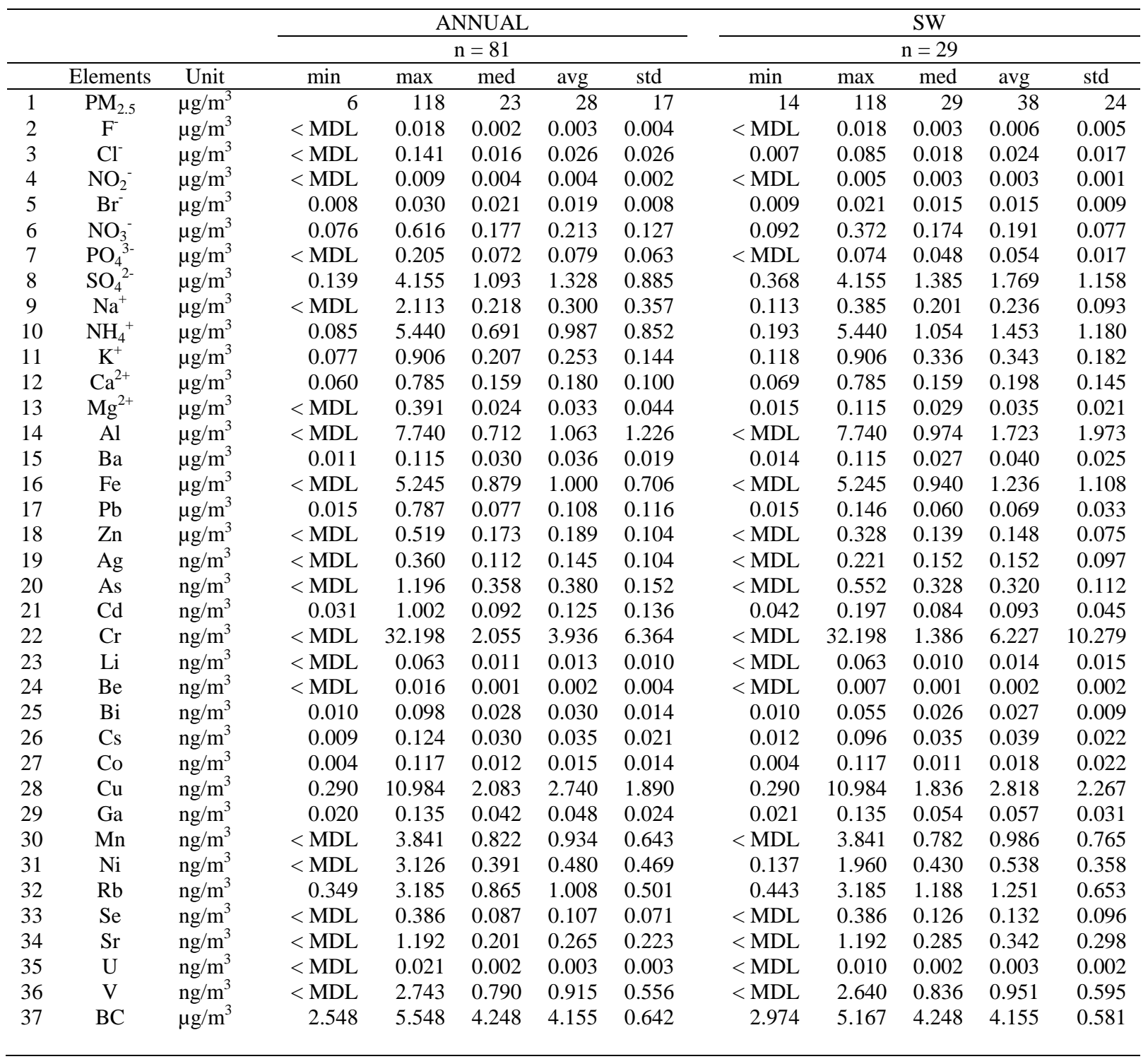

6 
4 Continuation of Table S2 (2)

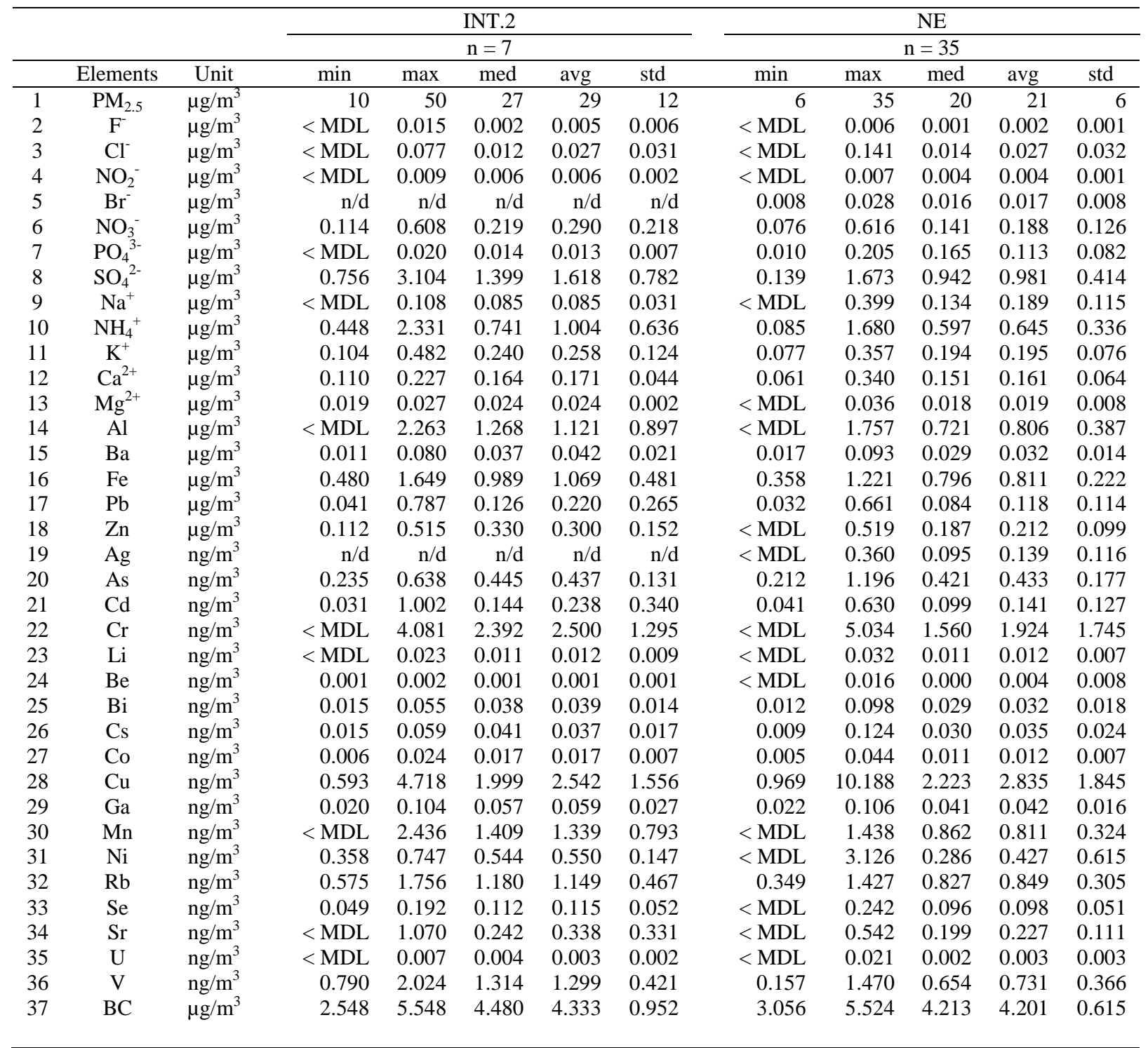




\begin{tabular}{|c|c|c|c|c|c|c|c|c|c|c|c|c|c|}
\hline & & & & & NT.1 & & & & & HAZE & & & \\
\hline & & & & & $=10$ & & & & & $n=11$ & & & \\
\hline & Elements & Unit & $\min$ & $\max$ & med & avg & std & $\min$ & $\max$ & med & avg & std & $\%$ Recovery \\
\hline 1 & $\mathrm{PM}_{2.5}$ & $\mu \mathrm{g} / \mathrm{m}^{3}$ & 14 & 39 & 22 & 23 & 8 & 40 & 118 & 54 & 61 & 24 & \\
\hline 2 & $\mathrm{~F}^{-}$ & $\mu \mathrm{g} / \mathrm{m}^{3}$ & $<\mathrm{MDL}$ & 0.011 & 0.004 & 0.004 & 0.004 & 0.003 & 0.018 & 0.011 & 0.011 & 0.006 & 105 \\
\hline 3 & $\mathrm{Cl}^{-}$ & $\mu \mathrm{g} / \mathrm{m}^{3}$ & 0.007 & 0.072 & 0.018 & 0.027 & 0.021 & 0.007 & 0.085 & 0.021 & 0.030 & 0.022 & 112 \\
\hline 4 & $\mathrm{NO}_{2}^{-}$ & $\mu \mathrm{g} / \mathrm{m}^{3}$ & $<$ MDL & 0.003 & 0.003 & 0.003 & 0.000 & 0.003 & 0.004 & 0.004 & 0.004 & 0.001 & 107 \\
\hline 5 & $\mathrm{Br}^{-}$ & $\mu \mathrm{g} / \mathrm{m}^{3}$ & 0.025 & 0.030 & 0.028 & 0.028 & 0.002 & $\mathrm{n} / \mathrm{d}$ & $\mathrm{n} / \mathrm{d}$ & $\mathrm{n} / \mathrm{d}$ & $\mathrm{n} / \mathrm{d}$ & $\mathrm{n} / \mathrm{d}$ & 99 \\
\hline 6 & $\mathrm{NO}_{3}^{-}$ & $\mu \mathrm{g} / \mathrm{m}^{3}$ & 0.170 & 0.559 & 0.215 & 0.267 & 0.125 & 0.092 & 0.372 & 0.194 & 0.219 & 0.142 & 131 \\
\hline 7 & $\mathrm{PO}_{4}{ }^{3-}$ & $\mu \mathrm{g} / \mathrm{m}^{3}$ & $<$ MDL & 0.114 & 0.075 & 0.080 & 0.014 & $\mathrm{n} / \mathrm{d}$ & $\mathrm{n} / \mathrm{d}$ & $\mathrm{n} / \mathrm{d}$ & $\mathrm{n} / \mathrm{d}$ & $\mathrm{n} / \mathrm{d}$ & 103 \\
\hline 8 & $\mathrm{SO}_{4}^{2-}$ & $\mu \mathrm{g} / \mathrm{m}^{3}$ & 0.269 & 2.631 & 0.949 & 1.057 & 0.698 & 0.719 & 4.155 & 2.276 & 2.403 & 1.235 & 106 \\
\hline 9 & $\mathrm{Na}^{+}$ & $\mu \mathrm{g} / \mathrm{m}^{3}$ & 0.343 & 2.113 & 0.367 & 0.637 & 0.611 & 0.233 & 0.233 & 0.233 & 0.233 & 0.000 & 110 \\
\hline 10 & $\mathrm{NH}_{4}{ }^{+}$ & $\mu \mathrm{g} / \mathrm{m}^{3}$ & 0.245 & 1.591 & 0.769 & 0.821 & 0.485 & 0.541 & 5.440 & 1.999 & 2.211 & 1.468 & 86 \\
\hline 11 & $\mathrm{~K}^{+}$ & $\mu \mathrm{g} / \mathrm{m}^{3}$ & 0.087 & 0.290 & 0.176 & 0.181 & 0.063 & 0.336 & 0.906 & 0.452 & 0.507 & 0.171 & 96 \\
\hline 12 & $\mathrm{Ca}^{2+}$ & $\mu \mathrm{g} / \mathrm{m}^{3}$ & 0.060 & 0.259 & 0.211 & 0.196 & 0.062 & 0.124 & 0.785 & 0.170 & 0.282 & 0.206 & 93 \\
\hline 13 & $\mathrm{Mg}^{2+}$ & $\mu \mathrm{g} / \mathrm{m}^{3}$ & 0.015 & 0.391 & 0.048 & 0.081 & 0.111 & 0.028 & 0.115 & 0.042 & 0.050 & 0.027 & 98 \\
\hline 14 & $\mathrm{Al}$ & $\mu \mathrm{g} / \mathrm{m}^{3}$ & 0.025 & 0.990 & 0.387 & 0.408 & 0.292 & $<\mathrm{MDL}$ & 7.740 & 2.050 & 2.788 & 2.564 & 36 \\
\hline 15 & $\mathrm{Ba}$ & $\mu \mathrm{g} / \mathrm{m}^{3}$ & 0.018 & 0.052 & 0.032 & 0.033 & 0.011 & 0.022 & 0.115 & 0.043 & 0.046 & 0.026 & \\
\hline 16 & $\mathrm{Fe}$ & $\mu \mathrm{g} / \mathrm{m}^{3}$ & 0.511 & 1.452 & 0.900 & 0.980 & 0.355 & 0.371 & 5.245 & 1.351 & 1.897 & 1.500 & 69 \\
\hline 17 & $\mathrm{~Pb}$ & $\mu \mathrm{g} / \mathrm{m}^{3}$ & 0.045 & 0.293 & 0.090 & 0.108 & 0.069 & 0.059 & 0.146 & 0.095 & 0.094 & 0.030 & 101 \\
\hline 18 & $\mathrm{Zn}$ & $\mu \mathrm{g} / \mathrm{m}^{3}$ & 0.050 & 0.256 & 0.142 & 0.137 & 0.065 & $<\mathrm{MDL}$ & 0.328 & 0.185 & 0.175 & 0.088 & 69 \\
\hline 19 & $\mathrm{Ag}$ & $\mathrm{ng} / \mathrm{m}^{3}$ & $<\mathrm{MDL}$ & 0.249 & 0.176 & 0.161 & 0.096 & $\mathrm{n} / \mathrm{d}$ & $\mathrm{n} / \mathrm{d}$ & $\mathrm{n} / \mathrm{d}$ & $\mathrm{n} / \mathrm{d}$ & $\mathrm{n} / \mathrm{d}$ & 74 \\
\hline 20 & As & $\mathrm{ng} / \mathrm{m}^{3}$ & 0.211 & 0.454 & 0.313 & 0.326 & 0.093 & 0.214 & 0.428 & 0.328 & 0.337 & 0.065 & 51 \\
\hline 21 & $\mathrm{Cd}$ & $\mathrm{ng} / \mathrm{m}^{3}$ & 0.034 & 0.135 & 0.075 & 0.080 & 0.033 & 0.051 & 0.197 & 0.139 & 0.130 & 0.047 & 47 \\
\hline 22 & $\mathrm{Cr}$ & $\mathrm{ng} / \mathrm{m}^{3}$ & $<\mathrm{MDL}$ & 10.545 & 2.865 & 3.370 & 3.063 & $<\mathrm{MDL}$ & 4.214 & 2.866 & 2.866 & 1.907 & 17 \\
\hline 23 & $\mathrm{Li}$ & $\mathrm{ng} / \mathrm{m}^{3}$ & $<\mathrm{MDL}$ & 0.030 & 0.014 & 0.015 & 0.006 & 0.002 & 0.063 & 0.010 & 0.021 & 0.021 & \\
\hline 24 & $\mathrm{Be}$ & $\mathrm{ng} / \mathrm{m}^{3}$ & 0.001 & 0.001 & 0.001 & 0.001 & 0.000 & 0.275 & 2.484 & 1.395 & 1.456 & 0.749 & \\
\hline 25 & $\mathrm{Bi}$ & $\mathrm{ng} / \mathrm{m}^{3}$ & 0.010 & 0.035 & 0.026 & 0.023 & 0.008 & 0.017 & 0.042 & 0.027 & 0.028 & 0.008 & \\
\hline 26 & Cs & $\mathrm{ng} / \mathrm{m}^{3}$ & 0.013 & 0.054 & 0.025 & 0.026 & 0.012 & 0.033 & 0.096 & 0.059 & 0.060 & 0.019 & 38 \\
\hline 27 & Co & $\mathrm{ng} / \mathrm{m}^{3}$ & 0.005 & 0.025 & 0.012 & 0.015 & 0.006 & 0.004 & 0.117 & 0.018 & 0.029 & 0.032 & 60 \\
\hline 28 & $\mathrm{Cu}$ & $\mathrm{ng} / \mathrm{m}^{3}$ & 1.468 & 4.383 & 2.040 & 2.323 & 1.002 & 1.517 & 6.840 & 3.264 & 3.491 & 1.962 & 65 \\
\hline 29 & $\mathrm{Ga}$ & $\mathrm{ng} / \mathrm{m}^{3}$ & 0.021 & 0.062 & 0.033 & 0.038 & 0.013 & 0.024 & 0.135 & 0.057 & 0.056 & 0.032 & \\
\hline 30 & $\mathrm{Mn}$ & $\mathrm{ng} / \mathrm{m}^{3}$ & 0.222 & 2.932 & 0.578 & 0.937 & 0.930 & $<\mathrm{MDL}$ & 3.841 & 1.113 & 1.388 & 1.064 & 72 \\
\hline 31 & $\mathrm{Ni}$ & $\mathrm{ng} / \mathrm{m}^{3}$ & $<\mathrm{MDL}$ & 0.928 & 0.402 & 0.456 & 0.283 & 0.196 & 1.960 & 0.425 & 0.588 & 0.496 & 65 \\
\hline 32 & $\mathrm{Rb}$ & $\mathrm{ng} / \mathrm{m}^{3}$ & 0.379 & 1.324 & 0.818 & 0.785 & 0.282 & 1.048 & 3.185 & 1.733 & 1.777 & 0.612 & 29 \\
\hline 33 & $\mathrm{Se}$ & $\mathrm{ng} / \mathrm{m}^{3}$ & $<\mathrm{MDL}$ & 0.087 & 0.058 & 0.055 & 0.026 & 0.066 & 0.386 & 0.203 & 0.207 & 0.085 & 42 \\
\hline 34 & $\mathrm{Sr}$ & $\mathrm{ng} / \mathrm{m}^{3}$ & $<\mathrm{MDL}$ & 0.228 & 0.133 & 0.132 & 0.058 & $<\mathrm{MDL}$ & 1.192 & 0.311 & 0.456 & 0.386 & 58 \\
\hline 35 & $\mathrm{U}$ & $\mathrm{ng} / \mathrm{m}^{3}$ & 0.001 & 0.004 & 0.002 & 0.002 & 0.001 & 0.515 & 9.890 & 2.730 & 3.820 & 2.829 & \\
\hline 36 & V & $\mathrm{ng} / \mathrm{m}^{3}$ & 0.240 & 2.743 & 1.035 & 1.186 & 0.832 & 0.612 & 2.640 & 0.841 & 1.024 & 0.574 & 64 \\
\hline 37 & $\mathrm{BC}$ & $\mu \mathrm{g} / \mathrm{m}^{3}$ & 3.091 & 4.799 & 3.591 & 3.865 & 0.676 & 4.202 & 5.167 & 4.529 & 4.611 & 0.332 & \\
\hline
\end{tabular}


1 Table S3. Seasonal results of Pearson correlation matrices between $\mathrm{PM}_{2.5}$ mass, chemical mass closure (CMC) components and positive matrix

2 factorisation (PMF) factors identified towards: a) meteorological parameters, and b) gaseous parameters. Remarks: $\mathrm{BC}=$ black carbon while

3 PMF factors were: factor $1=$ combustion of engine oil; factor $2=$ mineral dust; factor $3=$ mixed secondary inorganic aerosol (SIA) and biomass

4 burning; factor $4=$ mixed traffic and industrial; and factor $5=$ sea salt. For meteorological parameters, API is air pollution index; $\mathrm{T}=$

5 temperature; $\mathrm{RH}=$ relative humidity; $\mathrm{WS}=$ wind speed; and $\mathrm{WD}=$ wind direction. 


\begin{tabular}{|c|c|c|c|c|c|c|c|}
\hline a) & Variables & ANNUAL & SW & INT.2 & NE & INT.1 & HAZE \\
\hline \multirow[t]{12}{*}{ API } & $\mathrm{PM}_{25}$ mass & $\begin{array}{l}0.763 \\
\end{array}$ & 0.748 & 0.299 & 0.473 & 0.705 & 0.531 \\
\hline & $\mathrm{CMC} \mathrm{SO}_{4}^{2-}$ & 0.629 & 0.565 & 0.683 & 0.469 & 0.585 & 0.48 \\
\hline & $\mathrm{CMC} \mathrm{NO}_{3}^{-}$ & -0.172 & -0.35 & 0.37 & 0.079 & 0.623 & -0.175 \\
\hline & $\mathrm{CMC} \mathrm{NH}_{4}^{+}$ & 0.712 & 0.667 & 0.75 & 0.561 & 0.46 & 0.606 \\
\hline & CMC Sea salt & -0.087 & -0.294 & 0.214 & -0.088 & 0.547 & -0.102 \\
\hline & CMC Dust & 0.227 & 0.22 & -0.033 & -0.101 & 0.302 & -0.114 \\
\hline & CMC BC & 0.353 & 0.58 & 0.046 & 0.234 & 0.772 & 0.274 \\
\hline & PMF Factor 1 & 0.133 & -0.062 & -0.084 & 0.366 & -0.313 & 0.076 \\
\hline & PMF Factor 2 & 0.354 & 0.275 & -0.041 & -0.103 & 0.325 & 0.005 \\
\hline & PMF Factor 3 & 0.725 & 0.716 & 0.767 & 0.158 & 0.817 & 0.476 \\
\hline & PMF Factor 4 & -0.235 & 0.081 & -0.006 & -0.169 & 0.416 & 0.014 \\
\hline & PMF Factor 5 & 0.011 & -0.079 & -0.279 & -0.025 & 0.612 & -0.124 \\
\hline \multirow[t]{12}{*}{$\mathrm{T}$} & $\mathrm{PM}_{2.5}$ mass & 0.31 & 0.236 & 0.572 & 0.201 & 0.03 & -0.05 \\
\hline & $\mathrm{CMC} \mathrm{SO}_{4}{ }^{2-}$ & 0.149 & -0.063 & 0.089 & 0.414 & 0.112 & -0.547 \\
\hline & $\mathrm{CMC} \mathrm{NO}_{3}^{-}$ & 0.052 & 0.194 & 0.521 & -0.151 & -0.097 & 0.384 \\
\hline & $\mathrm{CMC} \mathrm{NH}_{4}^{+}$ & 0.145 & 0.046 & 0.087 & 0.176 & -0.542 & -0.259 \\
\hline & CMC Sea salt & 0.155 & 0.407 & 0.128 & 0.379 & 0.008 & 0.562 \\
\hline & CMC Dust & 0.576 & 0.641 & 0.692 & 0.581 & -0.216 & 0.761 \\
\hline & CMC BC & -0.023 & 0.188 & 0.689 & -0.421 & -0.079 & 0.489 \\
\hline & PMF Factor 1 & 0.063 & 0.022 & -0.393 & 0.083 & -0.11 & 0.086 \\
\hline & PMF Factor 2 & 0.599 & 0.673 & 0.703 & 0.464 & 0.734 & 0.789 \\
\hline & PMF Factor 3 & 0.236 & 0.229 & 0.342 & 0.177 & -0.184 & -0.094 \\
\hline & PMF Factor 4 & -0.189 & 0.122 & 0.709 & -0.28 & 0.403 & 0.089 \\
\hline & PMF Factor 5 & 0.213 & 0.313 & 0.14 & 0.163 & -0.405 & 0.687 \\
\hline \multirow[t]{12}{*}{ RH } & $\mathrm{PM}_{2.5}$ mass & -0.314 & -0.252 & -0.495 & -0.174 & 0.152 & 0.108 \\
\hline & $\mathrm{CMC} \mathrm{SO}_{4}{ }^{2-}$ & -0.054 & 0.186 & -0.007 & -0.352 & 0.148 & 0.646 \\
\hline & $\mathrm{CMC} \mathrm{NO}_{3}^{-}$ & -0.035 & -0.358 & -0.489 & 0.231 & 0.338 & -0.534 \\
\hline & $\mathrm{CMC} \mathrm{NH}_{4}^{+}$ & -0.112 & 0.011 & -0.01 & -0.113 & 0.504 & 0.332 \\
\hline & CMC Sea salt & -0.117 & -0.379 & -0.097 & -0.519 & 0.227 & -0.461 \\
\hline & CMC Dust & -0.615 & -0.716 & -0.697 & -0.543 & 0.437 & -0.742 \\
\hline & CMC BC & 0.022 & -0.189 & -0.64 & 0.396 & 0.228 & -0.472 \\
\hline & PMF Factor 1 & 0.056 & 0.186 & 0.439 & -0.116 & 0.139 & -0.039 \\
\hline & PMF Factor 2 & -0.652 & -0.738 & -0.778 & -0.547 & -0.6 & -0.759 \\
\hline & PMF Factor 3 & -0.226 & -0.192 & -0.275 & -0.111 & 0.053 & 0.229 \\
\hline & PMF Factor 4 & 0.212 & -0.219 & -0.655 & 0.334 & -0.567 & -0.205 \\
\hline & PMF Factor 5 & -0.22 & -0.353 & -0.131 & -0.219 & 0.6 & -0.57 \\
\hline
\end{tabular}

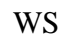

WD

\begin{tabular}{|c|c|c|c|c|c|c|}
\hline Variables & ANNUAL & SW & INT.2 & $\mathrm{NE}$ & INT.1 & HAZE \\
\hline $\mathrm{PM}_{25}$ mass & 0.274 & 0.164 & 0.245 & -0.03 & 0.192 & -0.446 \\
\hline $\mathrm{CMC} \mathrm{SO}_{4}{ }^{2-}$ & 0.174 & -0.047 & -0.125 & 0.224 & 0.208 & -0.692 \\
\hline $\mathrm{CMC} \mathrm{NO}_{3}^{-}$ & -0.113 & 0.161 & 0.378 & -0.429 & -0.055 & 0.719 \\
\hline $\mathrm{CMC} \mathrm{NH}_{4}^{+}$ & 0.14 & 0.015 & -0.103 & -0.034 & -0.421 & -0.53 \\
\hline CMC Sea salt & 0.013 & 0.007 & -0.035 & 0.241 & -0.195 & 0.111 \\
\hline CMC Dust & 0.385 & 0.421 & 0.588 & 0.408 & -0.154 & 0.43 \\
\hline CMC BC & -0.07 & 0.162 & 0.445 & -0.403 & -0.034 & -0.06 \\
\hline PMF Factor 1 & 0.084 & -0.066 & -0.599 & 0.107 & 0.557 & 0.104 \\
\hline PMF Factor 2 & 0.429 & 0.456 & 0.875 & 0.401 & 0.249 & 0.494 \\
\hline PMF Factor 3 & 0.213 & 0.107 & 0.182 & -0.186 & 0.228 & -0.678 \\
\hline PMF Factor 4 & -0.391 & 0.155 & 0.378 & -0.507 & 0.628 & 0.138 \\
\hline PMF Factor 5 & 0.132 & 0.048 & 0.128 & 0.144 & -0.267 & 0.306 \\
\hline $\mathrm{PM}_{25}$ mass & -0.131 & -0.181 & 0.409 & 0.056 & 0.047 & 0.413 \\
\hline $\mathrm{CMC} \mathrm{SO}_{4}{ }^{2-}$ & -0.054 & -0.006 & 0.047 & 0.083 & -0.029 & 0.162 \\
\hline $\mathrm{CMC} \mathrm{NO}_{3}^{-}$ & 0.002 & 0.17 & 0.471 & -0.226 & -0.124 & 0.451 \\
\hline $\mathrm{CMC} \mathrm{NH}_{4}^{+}$ & -0.105 & -0.012 & 0.034 & -0.029 & -0.524 & 0.319 \\
\hline CMC Sea salt & 0.096 & 0.064 & -0.19 & 0.309 & 0.145 & -0.039 \\
\hline CMC Dust & 0.014 & -0.085 & 0.625 & 0.406 & -0.699 & 0.197 \\
\hline CMC BC & -0.063 & -0.13 & 0.579 & -0.113 & -0.172 & 0.381 \\
\hline PMF Factor 1 & 0.205 & 0.581 & 0.129 & -0.147 & -0.15 & 0.677 \\
\hline PMF Factor 2 & -0.016 & -0.011 & 0.354 & 0.373 & -0.195 & 0.319 \\
\hline PMF Factor 3 & -0.161 & -0.257 & -0.045 & 0.144 & 0.633 & 0.259 \\
\hline PMF Factor 4 & 0.163 & -0.185 & 0.638 & 0.048 & 0.464 & 0.161 \\
\hline PMF Factor 5 & -0.067 & -0.152 & 0.339 & 0.121 & -0.312 & -0.011 \\
\hline $\mathrm{PM}_{2.5}$ mass & -0.212 & -0.246 & -0.733 & -0.052 & -0.051 & -0.178 \\
\hline $\mathrm{CMC} \mathrm{SO}_{4}{ }^{2-}$ & -0.136 & -0.133 & -0.422 & 0.025 & 0.001 & 0.236 \\
\hline $\mathrm{CMC} \mathrm{NO}_{3}^{-}$ & -0.03 & -0.03 & -0.414 & 0.037 & 0.079 & -0.257 \\
\hline $\mathrm{CMC} \mathrm{NH}_{4}^{+}$ & -0.092 & -0.188 & -0.335 & 0.207 & 0.618 & 0.012 \\
\hline CMC Sea salt & -0.106 & -0.274 & 0.109 & -0.093 & -0.251 & -0.213 \\
\hline CMC Dust & -0.228 & -0.353 & -0.296 & -0.265 & 0.551 & -0.391 \\
\hline CMC BC & -0.116 & -0.141 & -0.893 & -0.029 & 0.027 & -0.591 \\
\hline PMF Factor 1 & -0.037 & -0.084 & -0.291 & 0.191 & 0.53 & 0.064 \\
\hline PMF Factor 2 & -0.257 & -0.184 & -0.156 & -0.513 & -0.581 & -0.399 \\
\hline PMF Factor 3 & -0.207 & -0.227 & -0.227 & -0.265 & 0.047 & -0.097 \\
\hline PMF Factor 4 & -0.11 & -0.102 & -0.743 & -0.296 & -0.524 & -0.343 \\
\hline PMF Factor 5 & -0.05 & -0.128 & -0.816 & 0.144 & 0.338 & -0.101 \\
\hline
\end{tabular}


1 Continuation of Table S3 (2)

b)

\begin{tabular}{|c|c|c|c|c|c|c|}
\hline Variables & ANNUAL & SW & INT.2 & NE & INT.1 & HAZE \\
\hline $\mathrm{PM}_{2.5}$ mass & 0.471 & 0.687 & 0.713 & 0.488 & 0.654 & 0.749 \\
\hline $\mathrm{CMC} \mathrm{SO}_{4}{ }^{2-}$ & 0.455 & 0.579 & 0.793 & 0.428 & 0.582 & 0.412 \\
\hline $\mathrm{CMC} \mathrm{NO}_{3}^{-}$ & 0.171 & -0.292 & 0.334 & 0.296 & 0.687 & 0.014 \\
\hline $\mathrm{CMC} \mathrm{NH}_{4}^{+}$ & 0.462 & 0.664 & 0.711 & 0.576 & 0.177 & 0.674 \\
\hline CMC Sea salt & 0.02 & -0.273 & -0.085 & -0.482 & 0.626 & 0.107 \\
\hline CMC Dust & 0.06 & 0.232 & 0.001 & -0.285 & 0.331 & 0.205 \\
\hline CMC BC & 0.549 & 0.533 & 0.554 & 0.544 & 0.748 & 0.697 \\
\hline PMF Factor 1 & 0.206 & 0.134 & 0.722 & 0.498 & -0.167 & 0.306 \\
\hline PMF Factor 2 & 0.197 & 0.455 & -0.637 & -0.295 & 0.198 & 0.322 \\
\hline PMF Factor 3 & 0.302 & 0.612 & 0.42 & -0.083 & 0.072 & 0.6 \\
\hline PMF Factor 4 & 0.199 & 0.157 & 0.577 & 0.04 & -0.015 & 0.007 \\
\hline PMF Factor 5 & -0.025 & -0.163 & 0.438 & -0.167 & 0.694 & 0.01 \\
\hline $\mathrm{PM}_{2.5}$ mass & 0.298 & 0.535 & 0.427 & 0.433 & 0.378 & 0.449 \\
\hline $\mathrm{CMC} \mathrm{SO}_{4}{ }^{2-}$ & 0.198 & 0.191 & 0.674 & 0.531 & 0.239 & 0.803 \\
\hline $\mathrm{CMC} \mathrm{NO}_{3}^{-}$ & 0.079 & 0.032 & -0.055 & -0.087 & 0.345 & -0.413 \\
\hline $\mathrm{CMC} \mathrm{NH}_{4}^{+}$ & 0.241 & 0.343 & 0.553 & 0.521 & 0.627 & 0.685 \\
\hline CMC Sea salt & 0.121 & -0.014 & -0.21 & 0.333 & 0.237 & -0.332 \\
\hline CMC Dust & 0.115 & 0.193 & -0.467 & 0.207 & 0.316 & -0.532 \\
\hline CMC BC & 0.049 & 0.131 & 0.316 & -0.263 & 0.454 & -0.071 \\
\hline PMF Factor 1 & -0.287 & -0.605 & 0.796 & 0.118 & 0.04 & 0.358 \\
\hline PMF Factor 2 & -0.009 & 0.105 & -0.832 & -0.007 & -0.434 & -0.427 \\
\hline PMF Factor 3 & 0.304 & 0.597 & 0.204 & 0.383 & 0.703 & 0.538 \\
\hline PMF Factor 4 & 0.035 & 0.024 & 0.188 & -0.351 & 0.126 & -0.122 \\
\hline PMF Factor 5 & 0.147 & 0.113 & 0.638 & 0.305 & 0.509 & -0.379 \\
\hline $\mathrm{PM}_{2.5}$ mass & 0.324 & 0.141 & -0.25 & 0.654 & 0.627 & 0.445 \\
\hline $\mathrm{CMC} \mathrm{SO}_{4}{ }^{2-}$ & 0.345 & 0.201 & -0.559 & 0.407 & 0.67 & -0.017 \\
\hline $\mathrm{CMC} \mathrm{NO}_{3}^{-}$ & 0.03 & 0.009 & 0.041 & 0.061 & 0.523 & 0.193 \\
\hline $\mathrm{CMC} \mathrm{NH}_{4}^{+}$ & 0.31 & 0.267 & -0.442 & 0.46 & -0.404 & 0.353 \\
\hline CMC Sea salt & -0.028 & -0.057 & 0.611 & -0.191 & 0.261 & 0.279 \\
\hline CMC Dust & 0.189 & 0.207 & 0.331 & -0.022 & 0.031 & 0.425 \\
\hline CMC BC & 0.167 & 0.01 & -0.217 & 0.461 & 0.455 & 0.709 \\
\hline PMF Factor 1 & 0.38 & 0.301 & -0.349 & 0.436 & 0.395 & 0.276 \\
\hline PMF Factor 2 & 0.345 & 0.319 & 0.224 & 0.06 & 0.583 & 0.471 \\
\hline PMF Factor 3 & 0.181 & 0.108 & -0.26 & -0.008 & -0.168 & 0.375 \\
\hline PMF Factor 4 & -0.008 & 0.2 & -0.032 & 0.114 & 0.33 & 0.465 \\
\hline PMF Factor 5 & -0.026 & -0.082 & -0.581 & -0.211 & 0.32 & 0.267 \\
\hline
\end{tabular}

\begin{tabular}{|c|c|c|c|c|c|c|c|}
\hline & Variables & ANNUAL & SW & INT.2 & $\mathrm{NE}$ & INT.1 & HAZE \\
\hline \multirow[t]{12}{*}{$\mathrm{NO}_{\mathrm{X}}$} & $\mathrm{PM}_{2.5}$ mass & 0.058 & 0.112 & 0.8 & 0.38 & 0.588 & 0.192 \\
\hline & $\mathrm{CMC} \mathrm{SO}_{4}{ }^{2-}$ & 0.05 & 0.038 & 0.709 & 0.18 & 0.492 & -0.333 \\
\hline & $\mathrm{CMC} \mathrm{NO}_{3}^{-}$ & 0.284 & 0.085 & 0.545 & 0.295 & 0.609 & 0.429 \\
\hline & $\mathrm{CMC} \mathrm{NH}_{4}^{+}$ & 0.05 & 0.127 & 0.663 & 0.351 & 0.226 & 0.03 \\
\hline & CMC Sea salt & -0.063 & -0.04 & 0.005 & -0.527 & 0.472 & 0.307 \\
\hline & CMC Dust & -0.019 & 0.244 & 0.364 & -0.419 & 0.358 & 0.589 \\
\hline & CMC BC & 0.467 & 0.122 & 0.642 & 0.621 & 0.724 & 0.588 \\
\hline & PMF Factor 1 & 0.17 & 0.218 & 0.482 & 0.471 & -0.043 & 0.158 \\
\hline & PMF Factor 2 & 0.122 & 0.504 & -0.449 & -0.28 & 0.081 & 0.65 \\
\hline & PMF Factor 3 & -0.139 & 0.014 & 0.504 & -0.22 & 0 & 0.12 \\
\hline & PMF Factor 4 & 0.459 & 0.186 & 0.774 & 0.219 & -0.007 & 0.239 \\
\hline & PMF Factor 5 & -0.122 & 0.029 & 0.18 & -0.288 & 0.643 & 0.362 \\
\hline \multirow[t]{12}{*}{ NO } & $\mathrm{PM}_{2.5}$ mass & -0.262 & -0.309 & 0.701 & 0.086 & -0.126 & -0.285 \\
\hline & $\mathrm{CMC} \mathrm{SO}_{4}{ }^{2-}$ & -0.252 & -0.27 & 0.573 & -0.088 & -0.227 & -0.702 \\
\hline & $\mathrm{CMC} \mathrm{NO}_{3}^{-}$ & 0.185 & 0.177 & 0.353 & 0.126 & -0.006 & 0.432 \\
\hline & $\mathrm{CMC} \mathrm{NH}_{4}^{+}$ & -0.243 & -0.285 & 0.491 & 0.053 & 0.134 & -0.475 \\
\hline & CMC Sea salt & -0.062 & 0.215 & -0.073 & -0.475 & 0.013 & 0.434 \\
\hline & CMC Dust & -0.124 & 0.159 & 0.225 & -0.469 & -0.013 & 0.687 \\
\hline & CMC BC & 0.237 & -0.207 & 0.583 & 0.472 & 0.142 & 0.274 \\
\hline & PMF Factor 1 & 0.083 & 0.247 & 0.651 & 0.326 & -0.246 & 0.05 \\
\hline & PMF Factor 2 & -0.034 & 0.369 & -0.629 & -0.247 & -0.36 & 0.698 \\
\hline & PMF Factor 3 & -0.39 & -0.376 & 0.263 & -0.343 & -0.476 & -0.282 \\
\hline & PMF Factor 4 & 0.428 & 0.028 & 0.719 & 0.195 & -0.37 & 0.239 \\
\hline & PMF Factor 5 & -0.118 & 0.181 & 0.327 & -0.325 & 0.188 & 0.589 \\
\hline \multirow[t]{12}{*}{$\mathrm{NO}_{2}$} & $\mathrm{PM}_{2.5}$ mass & 0.473 & 0.528 & 0.851 & 0.711 & 0.874 & 0.599 \\
\hline & $\mathrm{CMC} \mathrm{SO}_{4}{ }^{2-}$ & 0.444 & 0.359 & 0.819 & 0.545 & 0.846 & 0.155 \\
\hline & $\mathrm{CMC} \mathrm{NO}_{3}^{-}$ & 0.287 & -0.052 & 0.751 & 0.454 & 0.778 & 0.269 \\
\hline & $\mathrm{CMC} \mathrm{NH}_{4}^{+}$ & 0.433 & 0.528 & 0.828 & 0.7 & 0.173 & 0.526 \\
\hline & CMC Sea salt & -0.041 & -0.309 & 0.112 & -0.408 & 0.583 & 0.069 \\
\hline & CMC Dust & 0.137 & 0.234 & 0.515 & -0.173 & 0.482 & 0.276 \\
\hline & CMC BC & 0.579 & 0.435 & 0.661 & 0.633 & 0.785 & 0.689 \\
\hline & PMF Factor 1 & 0.213 & 0.098 & 0.192 & 0.529 & 0.175 & 0.202 \\
\hline & PMF Factor 2 & 0.26 & 0.422 & -0.145 & -0.201 & 0.388 & 0.345 \\
\hline & PMF Factor 3 & 0.291 & 0.422 & 0.783 & 0.057 & 0.736 & 0.34 \\
\hline & PMF Factor 4 & 0.311 & 0.29 & 0.766 & 0.172 & 0.337 & 0.239 \\
\hline & PMF Factor 5 & -0.073 & -0.152 & -0.017 & -0.118 & 0.641 & 0.007 \\
\hline
\end{tabular}

2 Remarks: Values in bold are different from 0 with a significance level alpha $=0.05$ 
1 Table S4. Relative contribution from five identified sources for each HAZE episode.

Source contribution, $\mu \mathrm{g} \mathrm{m}^{-3}(\%)$

HAZE 2011 HAZE 2012

Factor 1: Combustion of engine oil

$$
\mathrm{n}=3 \quad \mathrm{n}=8
$$

Factor 2: Mineral dust

$1.17(2 \%) \quad 15.07(25 \%)$

Factor 3: Mixed SIA and biomass burning

$46.97(81 \%) \quad 33.15(56 \%)$

Factor 4: Mixed traffic and industrial

$1.12(2 \%) \quad 2.12(4 \%)$

Factor 5: Sea salt

$2.87(5 \%) \quad 5.28(9 \%)$

2 
1 Table S5. Pearson correlation matrix results between chemical mass closure (CMC)

2 components and positive matrix factorisation (PMF) factor for: a) HAZE 2011, and b) HAZE

32012 episodes. Remarks: SIA = secondary inorganic aerosol.

4 a) HAZE 2011

\begin{tabular}{|c|c|c|c|c|c|c|}
\hline & & & & & $\mathrm{CMC}$ & \\
\hline & PMF & $\mathrm{CMC}$ & $\mathrm{CMC}$ & & nss- & $\mathrm{CMC}$ \\
\hline Variables & Factor 3 & $\mathrm{SO}_{4}^{2-}$ & $\mathrm{NH}_{4}^{+}$ & $\mathrm{CMC} \mathrm{K}^{+}$ & $\mathrm{SO}_{4}^{2-}$ & SIA \\
\hline PMF Factor 3 & 1 & 0.899 & 0.873 & 0.915 & 0.899 & 0.888 \\
\hline $\mathrm{CMC} \mathrm{SO}_{4}{ }^{2-}$ & & 1 & 0.998 & 0.647 & 1.000 & 1.000 \\
\hline $\mathrm{CMC} \mathrm{NH}_{4}^{+}$ & & & 1 & 0.603 & 0.998 & 1.000 \\
\hline $\mathrm{CMC} \mathrm{K}^{+}$ & & & & 1 & 0.647 & 0.627 \\
\hline $\mathrm{CMC}$ nss- $\mathrm{SO}_{4}{ }^{2-}$ & & & & & 1 & 1.000 \\
\hline CMC SIA & & & & & & 1 \\
\hline
\end{tabular}

5 b) HAZE 2012

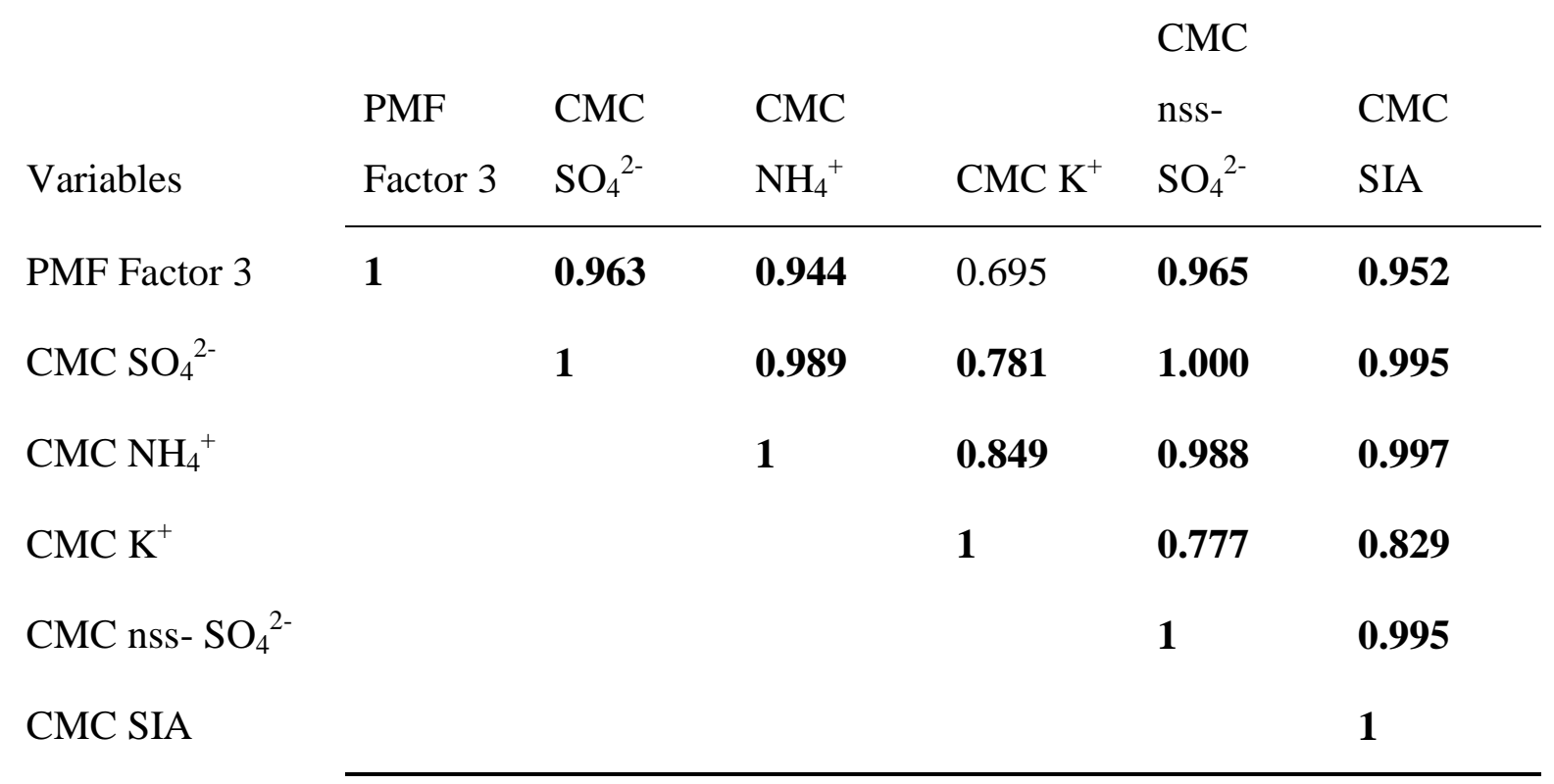

6 Values in bold are different from 0 with a significance level alpha=0.05. 
1 Table S6. HAZE episodes results for Pearson correlation matrices between PM2.5 mass, 2 chemical mass closure (CMC) components and positive matrix factorisation (PMF) factors 3 identified towards: a) meteorological parameters, and b) gaseous parameters. Remarks: $\mathrm{BC}=$ 4 black carbon while PMF factor details are as follows: factor 1= combustion of engine oil; 5 factor $2=$ mineral dust; factor $3=$ mixed secondary inorganic aerosol (SIA) and biomass 6 burning; factor $4=$ mixed traffic and industrial; and factor $5=$ sea salt. For meteorological 7 parameters, API is air pollution index; $\mathrm{T}=$ temperature; $\mathrm{RH}=$ relative humidity; $\mathrm{WS}=$ wind 8 speed; and $\mathrm{WD}=$ wind direction. 
a)

\begin{tabular}{lrr}
\hline \multirow{2}{*}{ Variables } & HAZE & HAZE \\
& 2011 & 2012 \\
\hline $\mathrm{PM}_{2.5}$ mass & 0.082 & 0.613 \\
$\mathrm{SO}_{4}{ }^{2-}$ & 0.491 & $\mathbf{0 . 7 3 6}$ \\
$\mathrm{NO}_{3}{ }^{-}$ & & -0.313 \\
$\mathrm{NH}_{4}{ }^{+}$ & 0.539 & 0.67
\end{tabular}

Sea salt $\quad 0.386 \quad-0.204$

Dust $\quad 0.145 \quad-0.298$

BC

Factor 1

Factor 2

Factor 3

$0.027 \quad 0.245$

Factor 4

$0.99 \quad 0.093$

$1-0.231$

$\begin{array}{ll}0.061 & 0.611\end{array}$

$-0.53 \quad-0.075$

$\mathrm{T}$

Factor 5

$\mathrm{PM}_{2.5}$ mass

$\mathrm{SO}_{4}{ }^{2-}$

$\mathrm{NO}_{3}{ }^{-}$

$\mathrm{NH}_{4}^{+}$

Sea salt

Dust

BC

Factor 1

Factor 2

Factor 3

Factor 4

Factor 5

RH

\begin{tabular}{lrr}
\hline $\mathrm{PM}_{2.5}$ mass & -0.715 & 0.317 \\
$\mathrm{SO}_{4}{ }^{2-}$ & -0.942 & $\mathbf{0 . 7 3 3}$ \\
$\mathrm{NO}_{3}{ }^{-}$ & & -0.54 \\
$\mathrm{NH}_{4}{ }^{+}$ & -0.959 & 0.637 \\
Sea salt & -0.896 & -0.49 \\
Dust & -0.758 & $\mathbf{- 0 . 7 7 9}$ \\
$\mathrm{BC}$ & -0.675 & -0.248 \\
Factor 1 & -0.841 & -0.214 \\
Factor 2 & $\mathbf{- 1}$ & $\mathbf{- 0 . 7 8 6}$ \\
Factor 3 & -0.7 & 0.568 \\
Factor 4 & -0.155 & 0.485 \\
Factor 5 & -0.759 & -0.567 \\
\hline
\end{tabular}

WS

\begin{tabular}{lrr}
\hline \multirow{2}{*}{ Variables } & HAZE & HAZE \\
& 2011 & 2012 \\
\hline $\mathrm{PM}_{2.5}$ mass & -0.921 & -0.432 \\
$\mathrm{SO}_{4}{ }^{2-}$ & -0.673 & -0.642 \\
$\mathrm{NO}_{3}{ }^{-}$ & & $\mathbf{0 . 7 5 9}$
\end{tabular}

$\begin{array}{lll}\mathrm{NH}_{4}{ }^{+} & -0.63 & -0.567\end{array}$

$\begin{array}{lll}\text { Sea salt } & -0.755 & 0.054\end{array}$

Dust $\quad-0.894 \quad 0.385$

$\begin{array}{lll}\text { BC } & -0.941 & -0.009\end{array}$

$\begin{array}{lll}\text { Factor } 1 & 0.176 & 0.17\end{array}$

$\begin{array}{lll}\text { Factor } 2 & \mathbf{- 1} & 0.413\end{array}$

$\begin{array}{lll}\text { Factor } 3 & -0.929 & -0.658\end{array}$

$\begin{array}{lll}\text { Factor } 4 & -0.972 & 0.063\end{array}$

\begin{tabular}{llrr} 
& Factor 5 & 0.309 & 0.234 \\
\cline { 2 - 4 } WD & $\mathrm{PM}_{2.5}$ mass & 0.6 & 0.427
\end{tabular}

$\begin{array}{lll}\mathrm{SO}_{4}{ }^{2-} & 0.88 & 0.316\end{array}$

$\mathrm{NO}_{3}{ }^{-} \quad 0.405$

$\begin{array}{lll}\mathrm{NH}_{4}{ }^{+} & 0.905 & 0.334\end{array}$

Sea salt $\quad 0.818 \quad-0.11$

$\begin{array}{lll}\text { Dust } & 0.65 & 0.095\end{array}$

$\begin{array}{lll}\text { BC } & 0.555 & 0.347\end{array}$

$\begin{array}{lll}\text { Factor } 1 & 0.913 & 0.713\end{array}$

$\begin{array}{lrr}\text { Factor } 2 & \mathbf{1} & 0.243\end{array}$

$\begin{array}{lll}\text { Factor } 3 & 0.583 & 0.284\end{array}$

$\begin{array}{lll}\text { Factor } 4 & 0.003 & 0.07\end{array}$

\begin{tabular}{lll} 
Factor 5 & 0.849 & -0.111 \\
\hline
\end{tabular}

\begin{tabular}{llll}
\cline { 3 - 4 } Rainfall & $\mathrm{PM}_{2.5}$ mass & $\mathbf{- 1}$ & -0.177
\end{tabular}

$\begin{array}{lll}\mathrm{SO}_{4}{ }^{2-} & \mathbf{- 1} & 0.064\end{array}$

$\mathrm{NO}_{3}{ }^{-}-0.244$

$\begin{array}{lll}\mathrm{NH}_{4}{ }^{+} & -1 & -0.013\end{array}$

Sea salt $\quad \quad \mathbf{- 1}-0.211$

Dust $\quad \mathbf{- 1}-0.389$

$\begin{array}{lll}\text { BC } & \mathbf{- 1} & -0.512\end{array}$

Factor $1 \quad 1$

Factor $2 \quad-0.44$

$\begin{array}{lll}\text { Factor } 3 & \mathbf{- 1} & 0.022\end{array}$

$\begin{array}{lll}\text { Factor } 4 & \mathbf{- 1} & 0.295\end{array}$

\begin{tabular}{lll} 
Factor 5 & $\mathbf{1}$ & -0.152 \\
\hline
\end{tabular} 


\begin{tabular}{|c|c|c|c|c|c|c|c|}
\hline b) & Variables & $\begin{array}{r}\text { HAZE } \\
2011\end{array}$ & $\begin{array}{r}\text { HAZE } \\
2012\end{array}$ & \multirow{13}{*}{$\mathrm{NO}_{\mathrm{X}}$} & Variables & $\begin{array}{r}\text { HAZE } \\
2011\end{array}$ & $\begin{array}{r}\text { HAZE } \\
2012\end{array}$ \\
\hline \multirow[t]{12}{*}{$\mathrm{CO}$} & $\mathrm{PM}_{2.5}$ mass & 0.541 & 0.907 & & $\mathrm{PM}_{2.5}$ mass & 0.577 & 0.319 \\
\hline & $\mathrm{SO}_{4}^{2-}$ & 0.843 & 0.797 & & $\mathrm{SO}_{4}^{2-}$ & 0.866 & -0.068 \\
\hline & $\mathrm{NO}_{3}{ }^{-}$ & & -0.18 & & $\mathrm{NO}_{3}^{-}$ & & 0.403 \\
\hline & $\mathrm{NH}_{4}{ }^{+}$ & 0.872 & 0.819 & & $\mathrm{NH}_{4}^{+}$ & 0.892 & 0.028 \\
\hline & Sea salt & 0.775 & -0.028 & & Sea salt & 0.802 & 0.227 \\
\hline & Dust & 0.594 & -0.014 & & Dust & 0.629 & 0.552 \\
\hline & $\mathrm{BC}$ & 0.494 & 0.691 & & $\mathrm{BC}$ & 0.532 & 0.604 \\
\hline & Factor 1 & 0.94 & 0.461 & & Factor 1 & 0.924 & 0.797 \\
\hline & Factor 2 & 1 & 0.111 & & Factor 2 & 1 & 0.677 \\
\hline & Factor 3 & 0.523 & 0.772 & & Factor 3 & 0.56 & 0.299 \\
\hline & Factor 4 & -0.069 & -0.334 & & Factor 4 & -0.025 & -0.548 \\
\hline & Factor 5 & 0.885 & -0.314 & & Factor 5 & 0.864 & 0.126 \\
\hline \multirow[t]{12}{*}{$\mathrm{O}_{3}$} & $\mathrm{PM}_{2.5}$ mass & -0.169 & 0.706 & \multirow[t]{12}{*}{ NO } & $\mathrm{PM}_{2.5}$ mass & 0.492 & -0.493 \\
\hline & $\mathrm{SO}_{4}^{2-}$ & 0.259 & 0.917 & & $\mathrm{SO}_{4}^{2-}$ & 0.811 & -0.778 \\
\hline & $\mathrm{NO}_{3}^{-}$ & & -0.391 & & $\mathrm{NO}_{3}^{-}$ & & 0.336 \\
\hline & $\mathrm{NH}_{4}{ }^{+}$ & 0.313 & 0.919 & & $\mathrm{NH}_{4}{ }^{+}$ & 0.842 & -0.715 \\
\hline & Sea salt & 0.145 & -0.326 & & Sea salt & 0.737 & 0.394 \\
\hline & Dust & -0.105 & -0.505 & & Dust & 0.546 & 0.625 \\
\hline & $\mathrm{BC}$ & -0.222 & 0.271 & & $\mathrm{BC}$ & 0.443 & -0.034 \\
\hline & Factor 1 & 0.924 & 0.451 & & Factor 1 & 0.958 & 0.142 \\
\hline & Factor 2 & 1 & -0.403 & & Factor 2 & 1 & 0.622 \\
\hline & Factor 3 & -0.189 & 0.856 & & Factor 3 & 0.473 & -0.71 \\
\hline & Factor 4 & -0.724 & 0.621 & & Factor 4 & -0.126 & -0.443 \\
\hline & Factor 5 & 0.967 & -0.5 & & Factor 5 & 0.91 & 0.52 \\
\hline \multirow[t]{12}{*}{$\mathrm{SO}_{2}$} & $\mathrm{PM}_{2.5}$ mass & 0.979 & 0.665 & \multirow[t]{12}{*}{$\mathrm{NO}_{2}$} & $\mathrm{PM}_{2.5}$ mass & 0.663 & 0.898 \\
\hline & $\mathrm{SO}_{4}^{2-}$ & 0.974 & 0.545 & & $\mathrm{SO}_{4}{ }^{2-}$ & 0.915 & 0.798 \\
\hline & $\mathrm{NO}_{3}^{-}$ & & -0.104 & & $\mathrm{NO}_{3}^{-}$ & & 0.059 \\
\hline & $\mathrm{NH}_{4}^{+}$ & 0.96 & 0.615 & & $\mathrm{NH}_{4}^{+}$ & 0.937 & 0.832 \\
\hline & Sea salt & 0.994 & 0.147 & & Sea salt & 0.862 & -0.193 \\
\hline & Dust & 0.99 & 0.141 & & Dust & 0.71 & -0.101 \\
\hline & $\mathrm{BC}$ & 0.966 & 0.652 & & $\mathrm{BC}$ & 0.621 & 0.694 \\
\hline & Factor 1 & 0.416 & 0.741 & & Factor 1 & 0.877 & 0.437 \\
\hline & Factor 2 & 1 & 0.263 & & Factor 2 & 1 & 0.037 \\
\hline & Factor 3 & 0.975 & 0.6 & & Factor 3 & 0.647 & 0.623 \\
\hline & Factor 4 & 0.664 & 0.004 & & Factor 4 & 0.085 & -0.117 \\
\hline & Factor 5 & 0.288 & 0.027 & & Factor 5 & 0.803 & -0.463 \\
\hline
\end{tabular}

1

2 Values in bold are different from 0 with a significance level alpha=0.05 

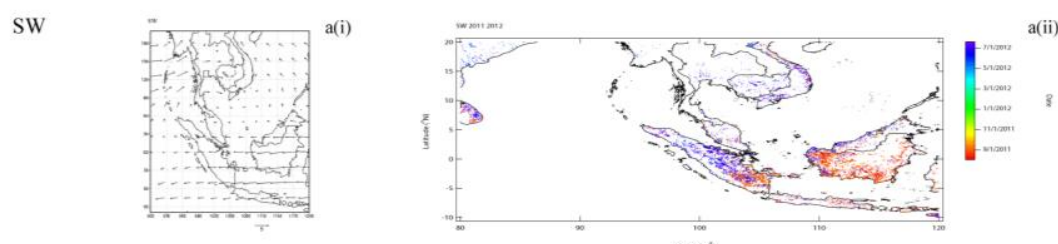

INT. 2
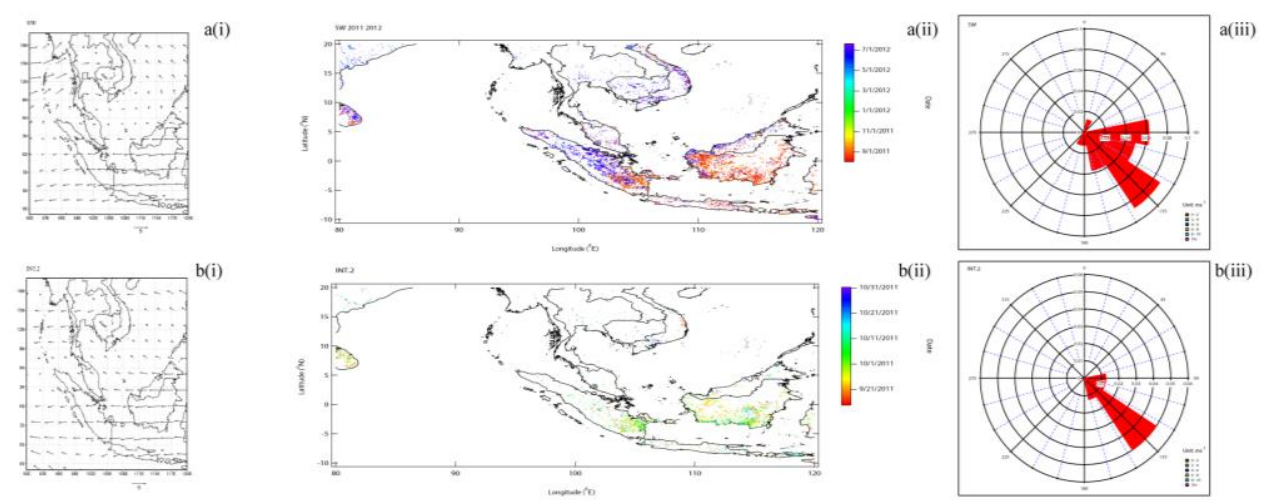

NE

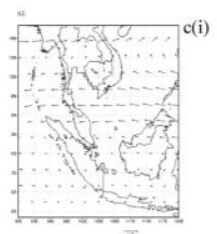

INT.1
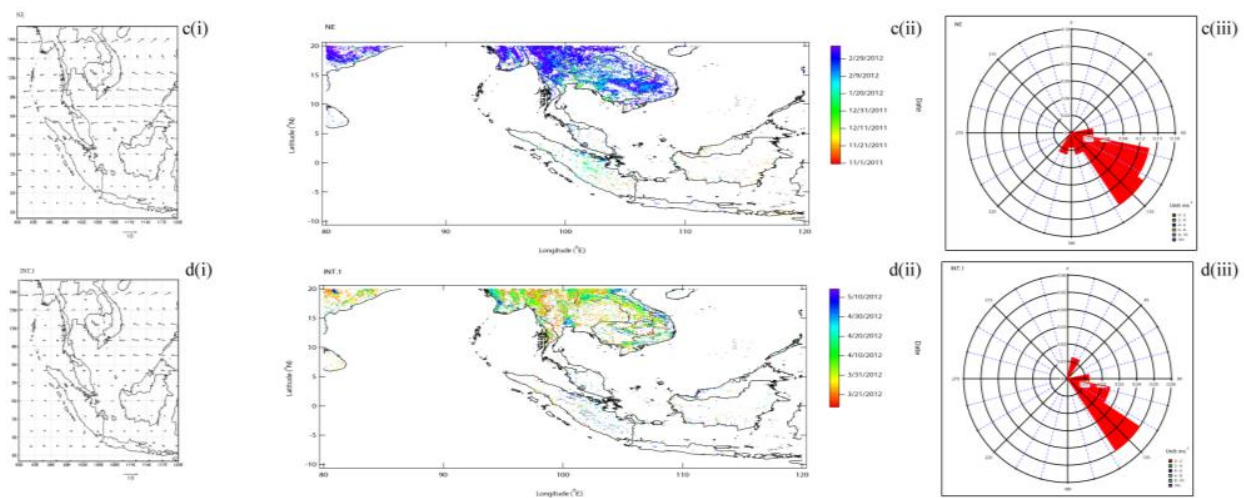

3 Figure S1. Seasonal plot for a) SW, b) INT.2, c) NE, and d) INT.1 pertaining to: i) regional 4 synoptic wind field plotted for $925 \mathrm{hPa}(500 \mathrm{~m})$; ii) biomass fire hotspot; and iii) wind rose 5 at the sampling site. 


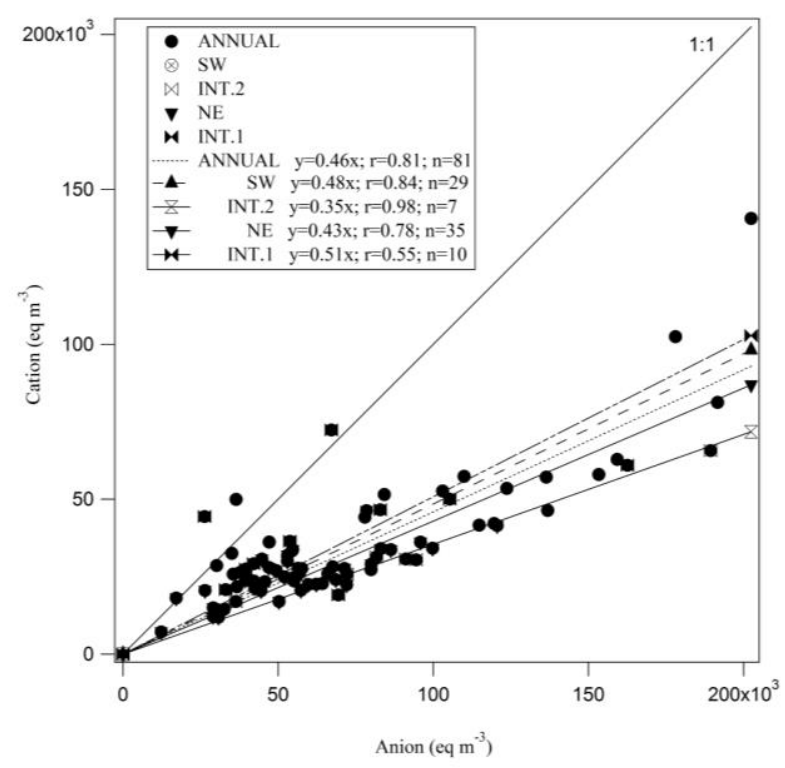

1

2 Figure S2. Correlations between cations and anions of $\mathrm{PM}_{2.5}$ on a seasonal and annual basis,

3 each with respective linear regression equations. The 1:1 line is given for comparison. 


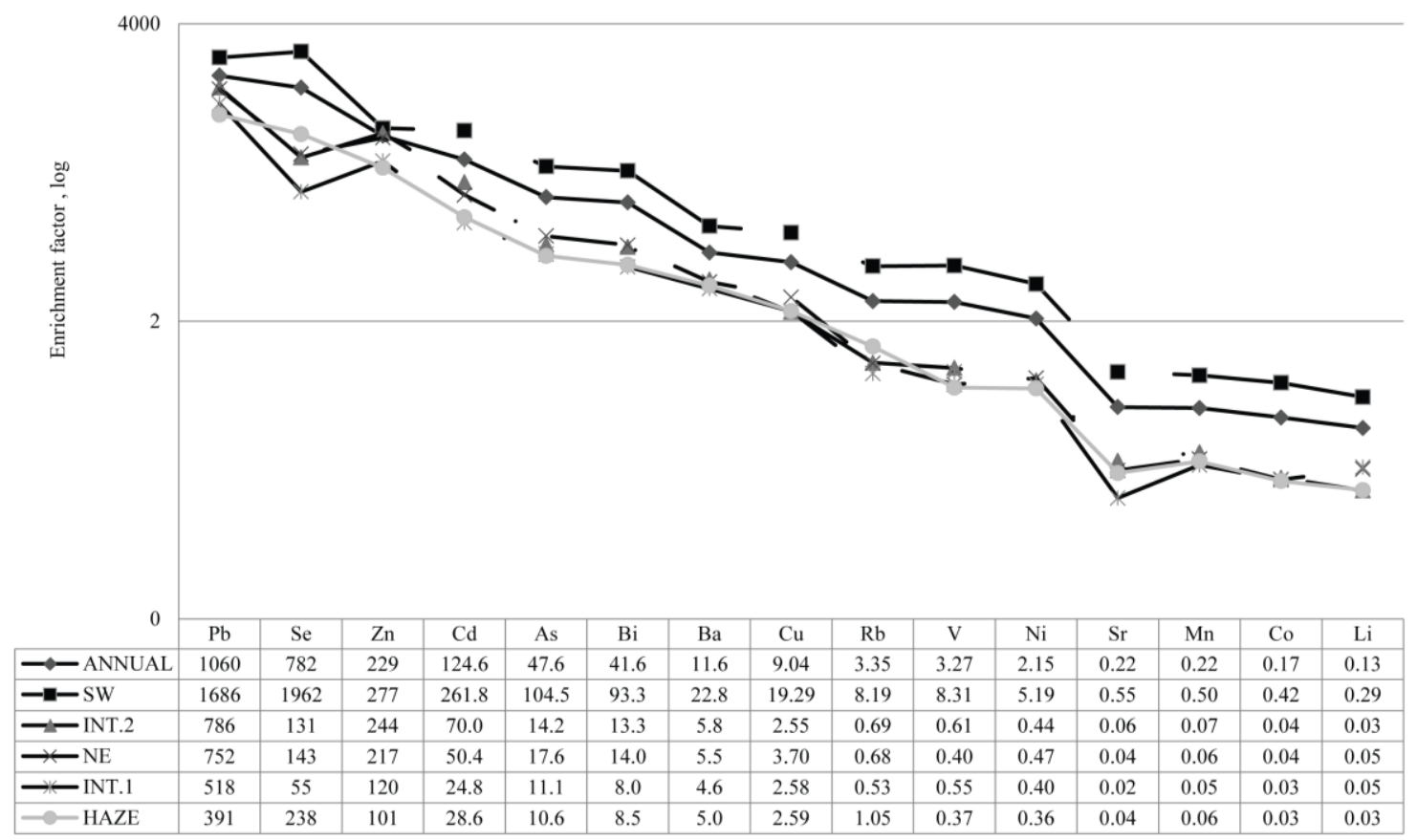

1

2 Figure S3. Seasonal variation of enrichment factor (EF) in the trace element of $\mathrm{PM}_{2.5}$. 

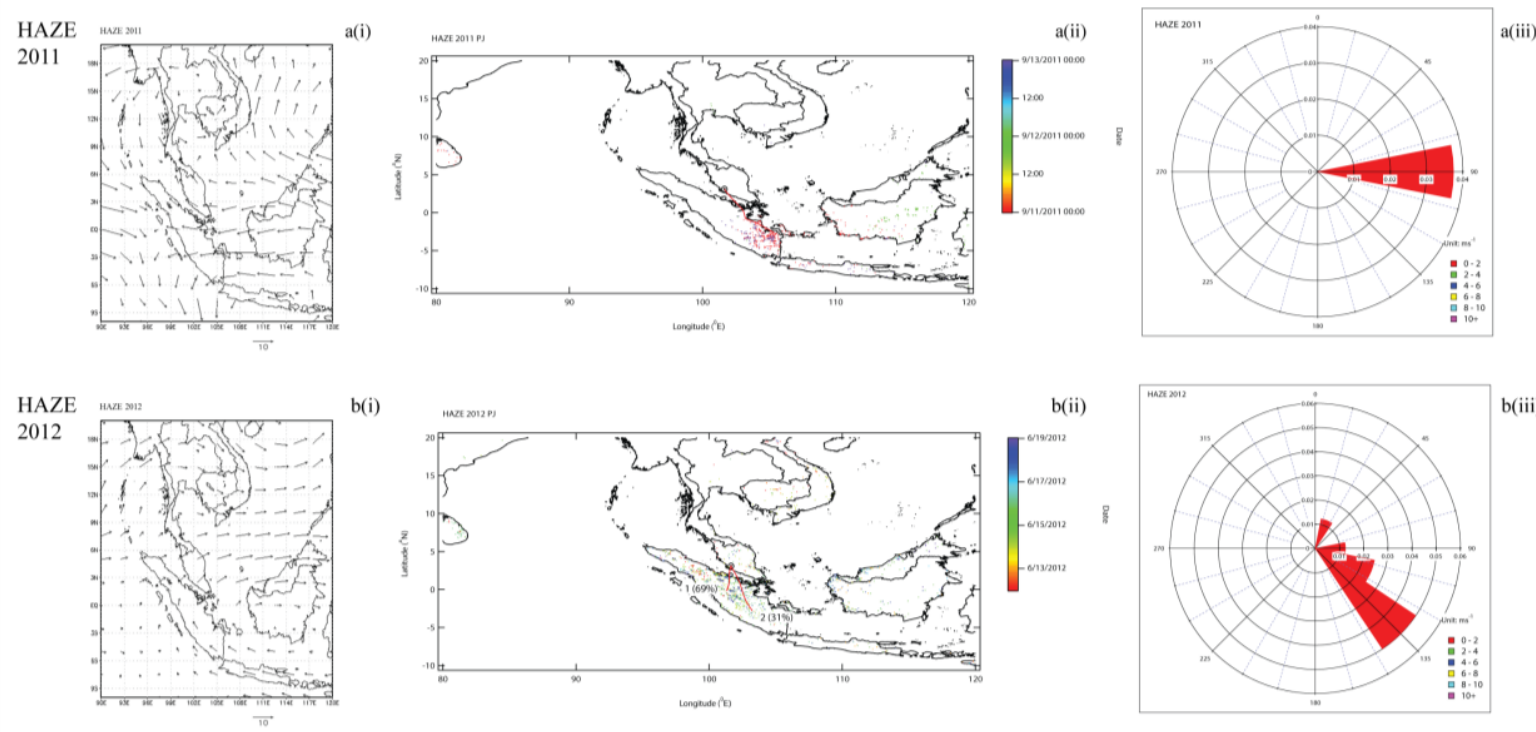

b(i)
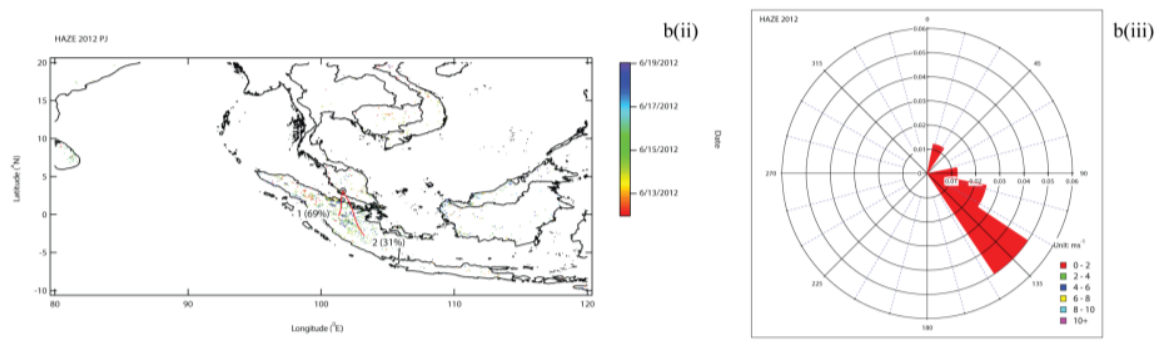

1

3 Figure S4. Haze episodes plot a) 2011 HAZE, and b) 2012 HAZE of i) synoptic wind field 4 plotted on $925 \mathrm{hPa}(500 \mathrm{~m})$; ii) biomass fire hotspot with $48 \mathrm{~h}$ backwards trajectories at the 5 releasing $925 \mathrm{hPa}(500 \mathrm{~m})$ with $6 \mathrm{~h}$ trajectory intervals; and iii) wind rose at the sampling 6 site. 


\section{Reference}

2

3 Squizzato, S., Masiol, M., Brunelli, A., Pistollato, S., Tarabotti, E., Rampazzo, G., and 4 Pavoni, B.: Factors determining the formation of secondary inorganic aerosol: a case study in 5 the Po Valley (Italy), Atmos. Chem. Phys., 13, 1927-1939, 10.5194/acp-13-1927-2013, 2013. 6 